\title{
Vortex formation of a Bose-Einstein condensate in a rotating deep optical lattice.
}

\author{
Akira Kato, Yuki Nakano, Kenichi Kasamatsu, and Tetsuo Matsui \\ Department of Physics, Kinki University, Higashi-Osaka, Osaka 577-8502, Japan
}

(Dated: August 6, 2018)

\begin{abstract}
We study the dynamics of vortex nucleation and lattice formation in a Bose-Einstein condensate in a rotating square optical lattice by numerical simulations of the Gross-Pitaevskii equation. Different dynamical regimes of vortex nucleation are found, depending on the depth and period of the optical lattice. We make an extensive comparison with the experiments by Williams et al. [Phys. Rev. Lett. 104, 050404 (2010)], especially focusing on the issues of the critical rotation frequency for the first vortex nucleation and the vortex number as a function of rotation frequency.

PACS numbers: $\quad$ 03.75.Lm, 03.75.Kk, 67.25.dk, 05.30.Jp
\end{abstract}

\section{INTRODUCTION}

Ultracold neutral atoms in an optical lattice (OL) are a particularly important system for studying a wide range of fundamental problems in condensed matter physics [1]. When an OL is rotated, the system mimics a lattice system of charged particles subject to a uniform magnetic field. This allows the development of versatile quantum simulators that can demonstrate various effects caused by a magnetic field such as quantum Hall effects [2, 3]. Recently, two experiments have been reported making use of a rotating OL to study quantized vortex dynamics in gaseous Bose-Einstein condensates (BECs) [4, 5]. In this system, the vortex pinning parameters can be controlled by changing conditions such as the amplitude, lattice constant, and rotation frequency of the OL. Moreover, the realization of a synthetic magnetic field [6], simulated by the Raman process between internal states of atoms, opens the possibility of studying a wide range of phenomena caused by artificial magnetic fields well under control.

Rotating BECs combined with a co-rotating OL exhibit a rich variety of vortex phases 7 [12], which have two competing length scales: the two lattice spacings, one for the OL and the other for the vortex lattice. The central role of the $\mathrm{OL}$ is to pin vortices at its maxima. Tung et al. 4] created a rotating square OL using a rotating mask, which provided a periodic pinning potential that was stationary in the rotating frame associated with the presence of vortices. They observed a structural crossover from a triangular to a square lattice of vortices with increasing potential amplitude of the OL. In a deep OL, the condensates are well localized at each potential minimum, so that the system enters a regime which can be regarded as a Josephson-junction array [10, 13, 14]. A bosonic Josephson-junction array under rotation (an analogue of a Josephson-junction array of superconductors under a magnetic field) can have characteristic vortex patterns with a unit-cell structure which depends on a filling factor, i.e., the number of vortices per unit cell of the periodic potential. This regime was recently demonstrated experimentally by Williams et al. [5].

Motivated by the experiment of Williams et al., we study the vortex lattice formation in a rotating BEC confined in a deep OL by numerical simulation of the Gross-Pitaevskii (GP) equation. We focus on the dynamics of vortices nucleation, which may depend on the properties of the OL, finding that the nucleation mechanism is greatly different from the surface instability in the case of a harmonically trapped rotating BEC 1517]. An interesting experimental observation is that the minimum rotation frequency needed to nucleate a single vortex decreases as the amplitude of the OL is increased, and eventually falls below the rotation frequency at which a single vortex at the center of a condensate is energetically stable [5]. This implies that vortices were nucleated locally at the lattice plaquette. However, our simulation reveals that this local nucleation does not occur for the experimental parameters used in Ref. [5]. We also show that inclusion of a Gaussian envelope of the lattice beams is important to explain the experimental observation of the vortex number dependence on the rotation frequency. A preliminary numerical study on vortex nucleation in an OL was reported by Yasunaga and Tsubota [18], but the case of a deep lattice limit was not discussed and sufficient understanding of the problem is still lacking.

The paper is organized as follows. In Sec. II] we describe the model of the problem and the parameter settings for our numerical simulations. We discuss the dynamics of the vortex lattice formation in a BEC under a rotating OL in Sec. III and make a detailed comparison with experiment in Sec. IV. Section $\mathrm{V}$ is devoted to a conclusion.

\section{FORMULATION OF THE PROBLEM}

We study the dynamics of a BEC trapped in an external potential $V(\mathbf{r})$ by employing the dissipative GP equation for the condensate wave function $\Psi(\mathbf{r}, t)[16,19$ 21]:

$$
\begin{aligned}
&(i-\gamma) \hbar \frac{\partial \Psi}{\partial t}=\left[-\frac{\hbar^{2}}{2 m} \nabla^{2}+V_{\text {ext }}(\mathbf{r})-\mu\right. \\
&\left.+g|\Psi(\mathbf{r}, t)|^{2}-\Omega L_{z}\right] \Psi(\mathbf{r}, t)
\end{aligned}
$$

Here, we take a frame rotating with frequency $\Omega$ around the $z$-axis. The total external potential $V_{\text {ext }}$ is given by 
the sum of the harmonic potential $V_{\text {ho }}$ and the OL $V_{\mathrm{OL}}$ :

$$
\begin{array}{r}
V_{\mathrm{ho}}=\frac{1}{2} m \omega_{\perp}^{2} r^{2}+\frac{1}{2} m \omega_{z}^{2} z^{2}, \\
V_{\mathrm{OL}}=V_{0}\left[\sin ^{2}(k x)+\sin ^{2}(k y)\right]
\end{array}
$$

with $r^{2}=x^{2}+y^{2}$; we shall consider another form of $V_{\mathrm{OL}}$ later (see Eq. (17)). . We have denoted that $L_{z}=-i \hbar\left(x \partial_{y}-y \partial_{x}\right)$ is the $z$-component of the angular momentum operator, $g=4 \pi \hbar^{2} a_{s} / m$ is the coupling constant with s-wave scattering length $a_{s}$, and $\gamma$ is the phenomenological dissipation parameter. The dissipation parameter is introduced to relax the system into the equilibrium configuration and is assumed to be $\gamma=0.03$ in the following [16, 19]; we shall discuss the $\gamma$-dependence of the results later.

To rewrite Eq. (11) into a dimensionless form, we use the characteristic scales of the harmonic potential as length $a_{\mathrm{ho}}=\sqrt{\hbar / m \omega_{\perp}}$, time $\omega_{\perp}^{-1}$, and energy $\hbar \omega_{\perp}$. Since we are concerned with the vortex pattern and dynamics in the two-dimensional (2D) $x-y$ plane, we reduce Eq. (1) into the $2 \mathrm{D}$ system. We assume that the profile of the wave function along the $z$-axis is approximately uniform by considering the system near the $z=0$ plane. Then, the wave function can be decomposed as $\Psi(x, y, z, t)=$ $\sqrt{N / R_{z}} \psi(x, y, t)$ with the normalization $\int d x d y|\psi|^{2}=1$, where $N$ is the total particle number and $R_{z}$ is a typical condensate size along the $z$-axis, taken as the ThomasFermi radius. The resulting dimensionless GP equation reads

$$
(i-\gamma) \frac{\partial \tilde{\psi}}{\partial \tilde{t}}=\left[-\frac{1}{2}\left(\partial_{\tilde{x}}^{2}+\partial_{\tilde{y}}^{2}\right)+\frac{\tilde{r}^{2}}{2}+\tilde{V}_{\mathrm{OL}}-\tilde{\mu}+\tilde{u}_{2 \mathrm{D}}|\tilde{\psi}|^{2}-\tilde{\Omega} \tilde{L}_{z}\right] \tilde{\psi}
$$

with $\tilde{V}_{\mathrm{OL}}=\tilde{V}_{0}\left(\sin ^{2} \tilde{k} \tilde{x}+\sin ^{2} \tilde{k} \tilde{y}\right)$ and the dimensionless coupling constant $\tilde{u}_{2 \mathrm{D}}=4 \pi N a_{s} / R_{z}$. Here, a dimensionless variable is denoted with a tilde, which shall be omitted in the following.

We use the following parameter values to reproduce the experimental setup of Williams et al. [5]: trapping frequencies $\left(\omega_{\perp}, \omega_{z}\right)=2 \pi(20.1,53.0) \mathrm{Hz}$, s-wave scattering length $a_{s}=5.61 \mathrm{~nm}$, and particle number $N=1.0 \times 10^{5}$ yield $a_{\mathrm{ho}}=2.4 \mu \mathrm{m}$ and $R_{z}=5.6 \mu \mathrm{m}$. Then, the coupling constant becomes $u_{2 \mathrm{D}} \approx 1000$. The spatial period $d=2 \mu \mathrm{m}$ of the OL used in the experiment corresponds to $d=\pi / k=0.83$ in our units. Hence, our free parameters are the potential depth $V_{0}$ in units of $\hbar \omega_{\perp}$ and the rotation frequency $\Omega$ in units of $\omega_{\perp}$. In the experiment [5], the range of $V_{0}$ is $100 \mathrm{~Hz} \leq V_{0} \leq 4000 \mathrm{~Hz}$, corresponding to $5 \leq V_{0} \leq 200$ in our units. Nucleation of vortices was observed by starting from a non-rotating condensate loaded into both a harmonic trap and a static OL, and then turning on the rotation of the OL. We use the ground state solution of Eq. (4) in the presence of both the non-rotating harmonic trap and the OL as the initial state of the simulations. The numerical scheme to solve Eq. (4) is a Crank-Nicholson method with spatial mesh $\Delta_{x, y}=0.05$ and time step $\Delta_{t}=0.0005$. Because the time development of Eq. (44) does not conserve the norm of the wave function for $\gamma \neq 0$, we treat the chemical potential $\mu$ as time-dependent and adjust it at each time step to ensure normalization, by calculating the correction $\Delta \mu=(\Delta t)^{-1} \ln \left[\int d^{2} r|\psi(t)|^{2} / \int d^{2} r|\psi(t+\Delta t)|^{2}\right]$ [22].

\section{DYNAMICS OF A ROTATING BEC IN AN OPTICAL LATTICE}

The observation of vortex nucleation and lattice formation of a harmonically trapped BEC [23] $\left(V_{0}=0\right)$ has been well reproduced by numerical simulations of the time-dependent GP equation [16, 17]. The simulation results clarified the interesting nonlinear dynamics where surface wave instability triggers vortex nucleation. We first show the overall nonlinear dynamics of vortex formation in a rotating BEC subject to an OL to reveal the mechanism of vortex nucleation. In this section, a rotation with $\Omega=0.6$ is suddenly started at $t=0$ of the simulations.

Figure 1 shows the time development of the condensate density for several values of the potential depth $V_{0}$. For the shallow OL $V_{0}=6$, the dynamics are similar to those without the OL, as shown in Fig. 1(a). First, the surface of the condensate becomes unstable and generates surface ripples that propagate along the surface. Then, the surface ripples gradually develop into vortex cores, and these vortices are pulled in toward the rotation axis to make a vortex lattice. The settled vortices are generally pinned by peaks of the OL. For $V_{0}=20$ [Fig. [1(b)], although vortices are also generated from the surface region, the condensate surface is disrupted to form density blobs rather than excited to form ripples. The vortices penetrate inside soon after the turn-on of the rotation. This is because quantized vortices accompany the density dips (vortex cores) so that they can easily penetrate into the condensate through the local density suppression caused by maxima of the OL. As the OL becomes much deeper, the system enters a regime of the Josephson-junction array. Here, fractions of the condensate are well localized at the potential minima and the overlap of the wave function between nearest-neighbor sites is very small. Then, the dynamics of the condensate density are completely frozen during the overall vortex nucleation process; only the phase is a dynamical degree of freedom. A typical example for $V_{0}=100$ is shown in Fig. 1(c). Even in this deep lattice, the vortices are nucleated from the periphery and settle very quickly as soon as the rotation is turned on.

The different behavior in the characteristic vortex nucleation time can be seen from the development of the free energy $F=E-\mu-\Omega\left\langle L_{z}\right\rangle$, shown in Fig. 1(d), where $E=\int d^{2} r \psi^{*}\left[-\left(\partial_{x}^{2}+\partial_{y}^{2}\right)+r^{2} / 2+V_{\mathrm{OL}}+u_{2 \mathrm{D}}|\psi|^{2}\right] \psi$. For a shallow lattice, the energy stays constant for a while just after the rotation is turned on. During this period, the vortices near the surface are prevented from entering. After some time, the energy rapidly decreases through 

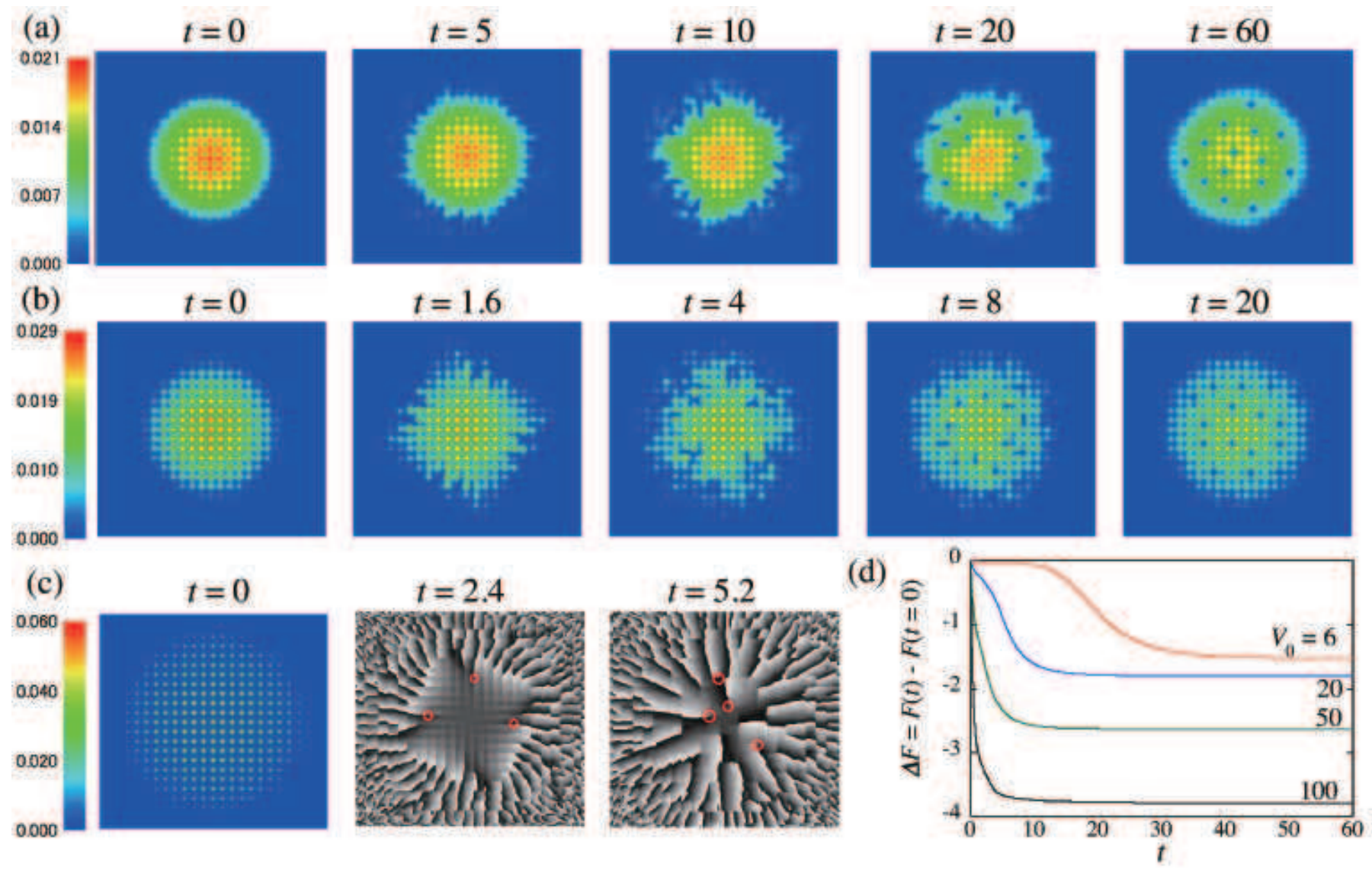

FIG. 1: (color online) Time development of the condensate density $|\psi|^{2}$ in the region $[-10,10] \times[-10,10]$ after the OL suddenly begins to rotate at $t=0$ with $\Omega=0.6$. The lattice constant of the OL is $d=0.83(k=1.2 \pi)$ and the height of the OL is $V_{0}=6$ (a), 20 (b), and 100 (c). In (c), the development of the phase $\theta=\arg \psi$ is also shown, where its value changes continuously from 0 (dark) to $2 \pi$ (bright). The vortices correspond to the ends of the branch cuts between the phases 0 and $2 \pi$; some of them are marked by circles. In (d), time evolutions of the free energy for several values of $V_{0}$ are plotted as the difference from the free energy at $t=0$. The physical units are $a_{\mathrm{ho}}=\sqrt{\hbar / m \omega_{\perp}}, \omega_{\perp}^{-1}$, and $\hbar \omega_{\perp}$ for length, time, and energy, respectively.

vortex penetration into the bulk. On the other hand, for a deep lattice, this period of constant energy cannot be seen. This means that the energy barrier for the vortex penetration is vanishingly small. Also, the vortices equilibrate soon due to the strong pinning effect of the OL. We checked that this qualitative feature of the decay process was not affected by the values of $\gamma$, which simply changed the decay time slightly for each numerical solution.

Yasunaga and Tsubota [18] found a different kind of vortex nucleation dynamic, in which an OL potential generates vortex-antivortex pairs inside the bulk region. We find that this nucleation dynamic occurs for a relatively large lattice spacing $d$. For $V_{0}=20$ but $k=0.3 \pi$ $(d=10 / 3)$, for example, a different dynamical feature from Fig. 1(b) arises as shown in Fig. 2, some vortices are created from the surface as usual, while others arise from vortex-antivortex pairs created by peaks of the OL moving inside the condensate. The phase profile at $t=0.6$ reveals the pair creation occurring at peaks of the OL [the bottom right panel of Fig. 2], because these vortices have circulations and anti-circulations of the phase. Then, the anti-vortices quickly disappear by migrating outward and combining with other vortices. However, some vortices remain in pairs in the condensate and form a vortex lattice together with vortices coming from the condensate surface. We find that similar dynamics occur for different values of $V_{0} \geq 20$. This nucleation mechanism is similar to that of dragging the superflow through an obstacle potential [24] or, more closely, stirring the condensate by a circularly moving narrow potential [25].

These different dynamical origins of vortex nucleation can be understood by noting that the density fluctuation is frozen for a deep OL. This condition can be obtained when the energy spacing $\sim(\pi \hbar / d)\left(2 V_{0} / m\right)^{1 / 2}$ of a single well of the OL under the harmonic approximation becomes larger than the typical interaction energy $\sim \mu$. Then we have $d<d_{c} \equiv 2 \pi \xi \sqrt{V_{0} / \mu}$ for the density to freeze, where $\xi=\hbar / \sqrt{2 m \mu}$ is the healing length. This estimation agrees fairly well with our numerical results 

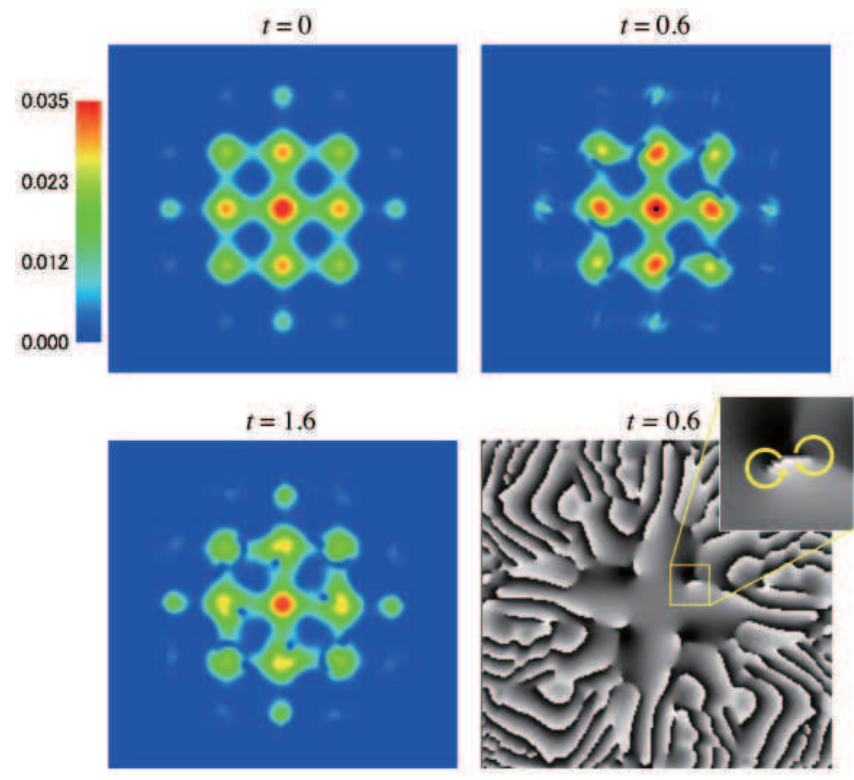

FIG. 2: (color online) Time development of the condensate density $|\psi|^{2}$ after the OL suddenly begins to rotate at $t=0$ with $\Omega=0.6 \omega_{\perp}$ for $V_{0}=20$ and $k=0.3 \pi\left(d=3.3 a_{\mathrm{ho}}\right)$ [compare to Fig. 1(b)]. The bottom right panel shows the phase profile and the inset is an enlarged view of a vortexantivortex pair.

because $d_{c} \sim 0.7$ in our parameters $(\mu \approx 83$ and $\xi \approx 0.1$ for $V_{0}=100$ and $\left.\Omega=0\right)$. The lattice spacing $d=2 \mu \mathrm{m}$ employed by Williams et al. corresponds to a regime in which the local vortex nucleation is not favorable because $d<d_{c} \sim 3 \mu \mathrm{m}$ for their parameters $V_{0}=2000 \mathrm{~Hz}$ and $\mu=500 \mathrm{~Hz}[5]$.

\section{COMPARISON WITH EXPERIMENTAL OBSERVATION}

Here, we compare our results with the experiment by Williams et al. [5]. Their significant observations are: (i) The minimum rotation frequency of the first vortex nucleation decreased with increasing $V_{0}$ and falls below $2 \pi \times 1 \mathrm{~Hz}\left(\Omega=0.05\right.$ in our physical units) above $V_{0} \simeq$ $1500 \mathrm{~Hz}\left(V_{0}=75\right.$ in our physical units). (ii) For the deep lattice $\left(V_{0}>\mu\right)$, the equilibrium vortex number increased linearly as a function of the rotation frequency $\Omega$. This point (ii) indicated that the condensate radius $R_{\perp}$ was not affected by the centrifugal expansion incidental to a rotating BEC in a harmonic potential, see Eq. (6) below.

In Fig. 3 we show the vortex number $N_{\mathrm{v}}$ in the equilibrium state as a function of the rotation frequency $\Omega$ for several values of $V_{0}$. This result was obtained by the dynamical simulation shown in the previous section with a sufficiently long time evolution to ensure the equilibration. We can see several features: (i) Naturally, the vortex number $N_{\mathrm{v}}$ increases monotonically with increas-

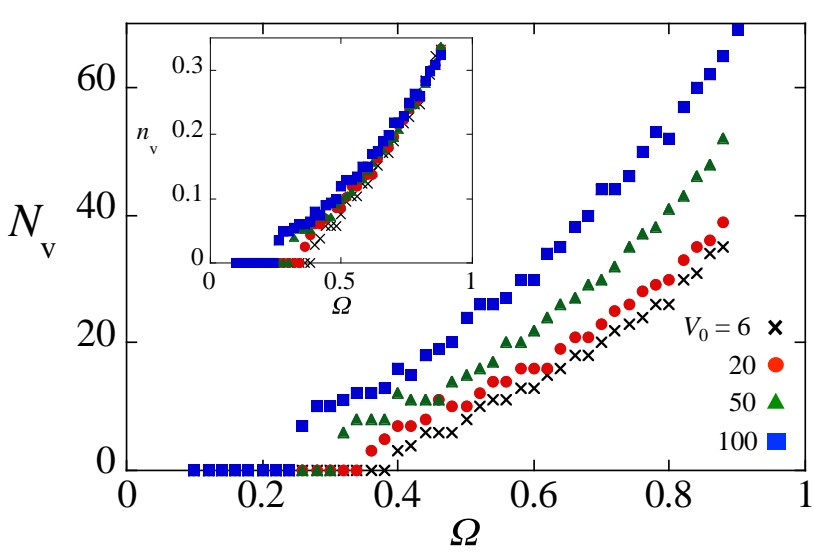

FIG. 3: (color online). Equilibrium vortex number as a function of $\Omega$ for several values of the potential depth $V_{0}$. We count the number of vortices within the radius determined by peripheral sites at which the density peak has $10 \%$ of the central peak density. The inset shows the behavior of the vortex number density taken by the condensate radius at $\Omega=0$, i.e. $n_{\mathrm{v}}=N_{\mathrm{v}} / \pi R_{\perp}(\Omega=0)^{2}$.

ing $\Omega$. In contrast to the experiment, it grows faster than a linear function for all $V_{0}$. (ii) In contrast with Ref. [5], the vortex number $N_{\mathrm{v}}$ also increases with increasing $V_{0}$ even at the same rotation frequency. This is because the tight confinement by the deep lattice effectively enhances the condensate radius $R_{\perp}$ under the condition of fixed 2D particle number [27]. The vortex number thus increases with the radius because the lattice spacing of vortices is fixed by $\Omega$. However, this property is irrelevant to the $\Omega$-dependence of $N_{\mathrm{v}}$, because this $2 \mathrm{D}$ artifact only affects the radius at $\Omega=0$. As shown in the inset of Fig. 3, when we plot the vortex density taken by the condensate radius at $\Omega=0$, i.e. $n_{\mathrm{v}}=N_{\mathrm{v}} / \pi R_{\perp}(\Omega=0)^{2}$, all the plots are almost coincident. This also supports the feature (i), namely, the scaling of $N_{\mathrm{v}}$ on $\Omega$ is independent of $V_{0}$. (iii) The critical rotation frequency for the first vortex nucleation is decreased as $V_{0}$ increases. However, it is still above $\Omega=0.2$ even for the very deep lattice $V_{0}=100$ in contrast with the experiment [5]. In the following, we consider these issues in more detail.

\section{A. Critical rotation frequency}

Our first concern is the critical rotation frequency of the vortex nucleation. To support our results, we calculate the thermodynamic critical frequency

$$
\Omega_{c}=\frac{E_{1}-E_{0}}{\left\langle L_{z}\right\rangle}
$$

that ensures thermodynamic stability of the stationary state with a single vortex at the origin [26]. Here, $E_{1}$ and $E_{0}$ are the total energy for the single vortex state 


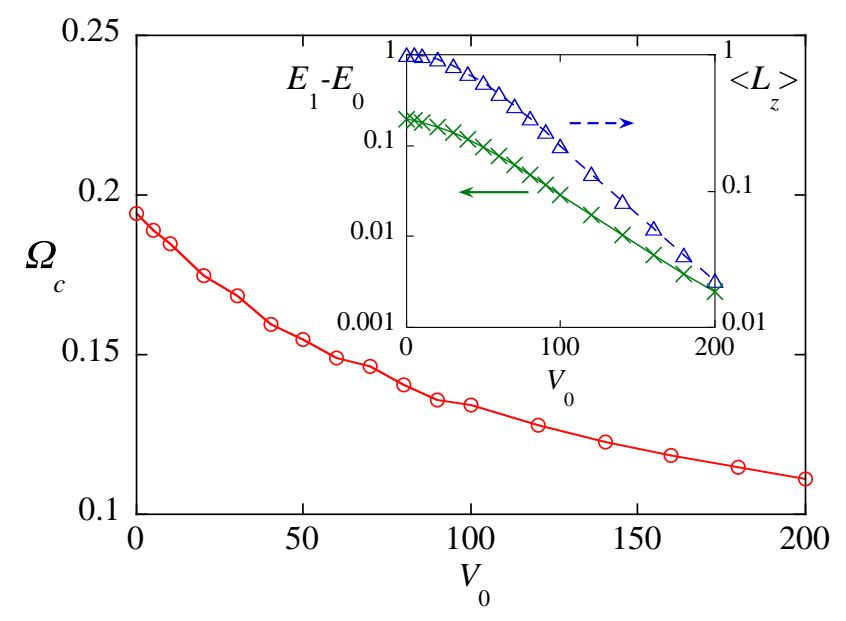

FIG. 4: (color online) Thermodynamic critical frequency $\Omega_{c}$ for the single vortex state as a function of the potential depth $V_{0}$. The inset shows the corresponding energy difference (numerator of the right-hand side of Eq. (5)) and the mean angular momentum per atom (denominator of the right-hand side of Eq. (5) ).

and the non-vortex state, respectively, and $\left\langle L_{z}\right\rangle$ is the mean angular momentum of the single vortex state. Since our OL of Eq. (3) has a sine form which has a minimum at the origin, we replace it with a cosine form with a maximum at the origin, which is suitable for calculating the energy of the single vortex state pinned at the center. Note that this critical frequency is not relevant for the actual event of vortex nucleation seen in Fig. 1, because the surface instability dominates the vortex nucleation. Nevertheless, we can obtain a lower bound of the critical rotation frequency because the critical rotation frequency $\Omega_{\text {sur }}$ associated with the surface instability is typically larger than $\Omega_{c}$ of Eq. (5) [15 17].

Figure 4 shows that the critical rotation frequency $\Omega_{c}$ decreases very slowly with $V_{0}$ and does not fall below 0.05 even at $V_{0}=200$. We find that, although the energy difference $E_{1}-E_{0}$ decreases exponentially with increasing $V_{0},\left\langle L_{z}\right\rangle$ also decreases together so that their ratio $\Omega_{c}$ does not vanish. For sufficiently large $V_{0}$, there is little energy cost for a vortex to be positioned at the potential maximum, because an almost zero density region extends between the well separated condensate islands at the potential maxima. Correspondingly, the overlap of the wave functions between nearest neighbor sites decreases significantly, which leads to the suppression of the current flowing between the sites. This reduces the atoms contributing to the mean angular momentum.

Thus, the dynamical simulations discussed in Sec. [II] cannot account for the observation by Williams et al. [5], because the vortices always nucleate from outside of the surface and are prevented from invading into the bulk for $\Omega<\Omega_{c}<\Omega_{\text {sur }}$. Since no vortices were observed in a static OL $(\Omega=0)$ [28], we can exclude the possibility of vortex nucleation due to thermal activation like the Berezenskii-Kosterlitz-Thouless mechanism [29] or interference between uncorrelated BECs [30]. Once vortices nucleate, they are expected to have a long life time because of the strong pinning effect of the OL, even if they are thermodynamically unstable. A more detailed study including the effect of the strong fluctuation of the OL [5], or applying a more elaborate model such as the projected GP equation [31] or stochastic GP equation 32 to this problem is a challenge for future study.

\section{B. Rotation frequency vs. vortex number}

The second concern is the linear dependence of the vortex number $N_{\mathrm{v}}$ as a function of the rotation frequency $\Omega$. For a superfluid rotating in a rigid container with radius $R$, the vortex number $N_{\mathrm{v}}$ can be determined by Feynman's relation $N_{\mathrm{v}}=m \Omega R^{2} / \hbar[33]$. In the ThomasFermi limit and if we smooth out the periodicity of the density caused by the OL, the radius of a harmonically trapped condensate under rotation is given by $R_{\perp}(\Omega)=$ $R_{\perp}(\Omega=0)\left[1-\left(\Omega / \omega_{\perp}\right)^{2}\right]^{-\nu}$ with $\nu=3 / 10$ for the $3 \mathrm{D}$ case and $1 / 4$ for the $2 \mathrm{D}$ case [26]. Therefore, the radius expands as $\Omega \rightarrow \omega_{\perp}$ and the vortex number diverges as

$N_{\mathrm{v}}=\frac{m \Omega}{\hbar} R_{\perp}(\Omega)^{2}=\frac{m \Omega}{\hbar} R_{\perp}(\Omega=0)^{2}\left[1-\left(\frac{\Omega}{\omega_{\perp}}\right)^{2}\right]^{-2 \nu}$.

Contrary to this, Williams et al. observed that for a deeper lattice the vortex number increased linearly with increasing $\Omega$ and did not diverge at $\Omega=\omega_{\perp}$; the vortex number was nearly coincident with the prediction of Eq. (6) without the factor $\left[1-\left(\Omega / \omega_{\perp}\right)^{2}\right]^{-2 \nu}$, that is $N_{\mathrm{v}} \propto \Omega$. This result was expected to be caused by the effect of the OL, where the radial expansion may be suppressed by the small tunneling rate at the peripheral lattice sites because the number of atoms decreases there.

We plot the vortex number taken from the numerical simulations as well as Eq. (6) in Fig. 5. To this end, we regard the radius $R_{\perp}(\Omega=0)$ as a fitting parameter because it is a somewhat arbitrary value for a trapped BEC and our interest here is the scaling property of $N_{\mathrm{v}}$ with respect to $\Omega$. For $V_{0}=20$, where the OL plays a minor contribution, the vortex number can be well fitted by Eq. (6), consistent with the experimental observation [5].

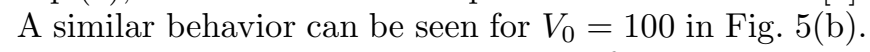
Thus, we do not see a linear behavior of the vortex number as observed experimentally.

In order to study the effect of an OL in more detail, we need a more realistic model of an OL. Here, we take into account the change in $V_{\mathrm{OL}}$ due to the Gaussian envelope of the lattice beams as

$$
V_{\mathrm{OL}}=-V_{0} e^{-2 r^{2} / w^{2}}\left[\cos ^{2}(k x)+\cos ^{2}(k y)\right]
$$

with beam waist $w=69 \mu \mathrm{m}$ [34]. Since the Gaussian envelope behaves as $1-2 r^{2} / w^{2}+\mathcal{O}\left(r^{4} / w^{4}\right)$, it leads to an increase in the frequency of the radial harmonic trap 

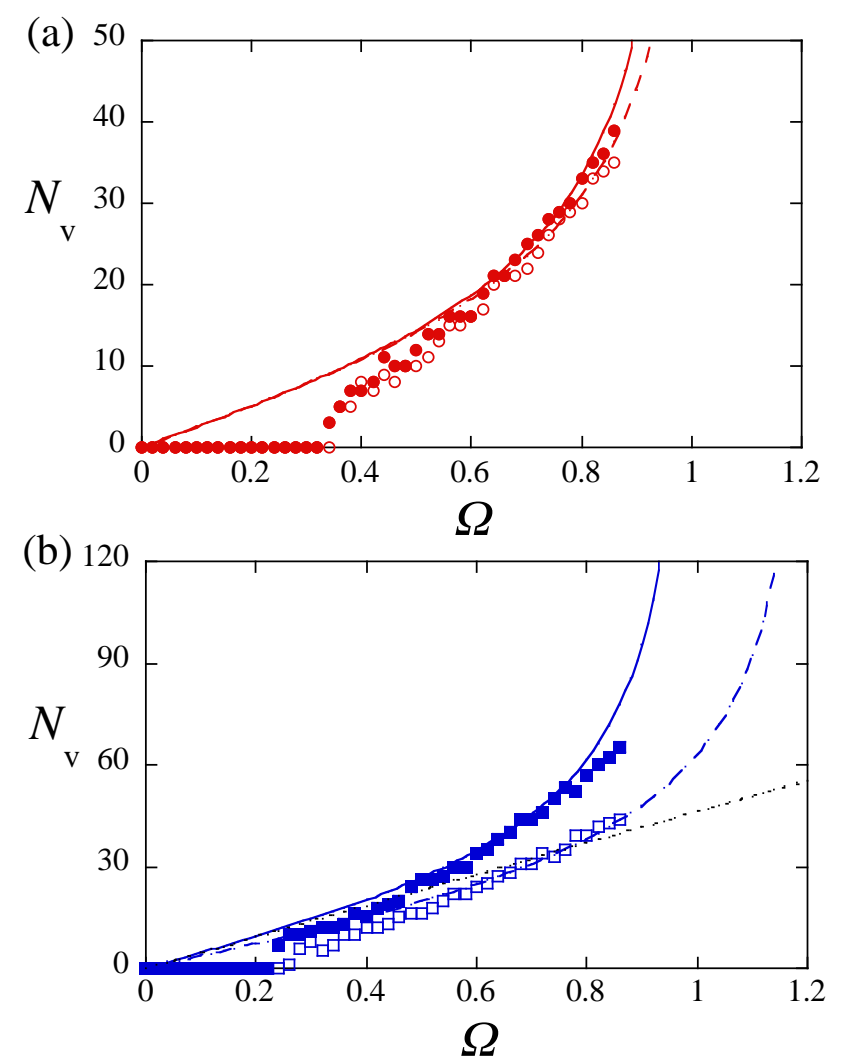

FIG. 5: (color online). Comparison of the vortex number with Feynman's rule for $V_{0}=20$ (a) and $V_{0}=100$ (b). The numerical results are plotted by filled symbols for the OL Eq. (3) and by empty symbols for the OL with a Gaussian envelope Eq. (77). The solid and dashed curves correspond to Eq. (6) and Eq. (8), respectively, with $\nu=1 / 4$. The fitting parameter $R_{\perp}(\Omega=0)$ is 5.0 for both curves in (a) and 6.8 and 6.1 for the solid and dashed curves in (b). The dotted line in (b) represents $N_{\mathrm{v}}=R_{\perp}(\Omega=0)^{2} \Omega$ with $R_{\perp}(\Omega=0)=6.8$.

as $\sqrt{\omega_{\perp}^{2}+4 V_{0} / m w^{2}}$. Under this modified potential, although the dynamical features of vortex nucleation are not altered, we can observe a significant reduction of $N_{\mathrm{v}}$ for large values of $V_{0}$, as shown by the empty symbols in Fig. 5. This reduction is caused by two contributions. The increase in the radial trap frequency makes the initial radius $R_{\perp}(\Omega=0)$ smaller than that without the Gaussian envelope. We found no visible change of
$R_{\perp}(\Omega=0)$ for $V_{0}=20$, while $R_{\perp}(\Omega=0)$ becomes about $10 \%$ smaller for $V_{0}=100$. Also, the Gaussian envelope suppresses the centrifugal expansion at $\Omega \rightarrow \omega_{\perp}$, which allows the condensate radius to remain finite even for $\Omega>\omega_{\perp}$, as observed in the experiment [5]. If we take up to the second order of $R_{\perp} / w$, Eq. (6) is modified as

$$
N_{\mathrm{v}}=\frac{m \Omega}{\hbar} R_{\perp}(\Omega=0)^{2}\left[\frac{1-\left(\frac{\Omega}{\omega_{\perp}}\right)^{2}+\frac{4 V_{0}}{m \omega_{\perp}^{2} w^{2}}}{1+\frac{4 V_{0}}{m \omega_{\perp}^{2} w^{2}}}\right]^{-2 \nu},
$$

which is plotted as the dashed curves in Fig. 5. Although only a minor modification occurs for small $V_{0}$, it is easy to see why the linear-like behavior was observed in the range $0<\Omega<1$ for large $V_{0}$. Our numerical results actually appear to follow the line of the linear scaling, as seen in Fig. 5(b).

\section{CONCLUSION}

We discuss the dynamics of vortex lattice formation in a BEC subject to a rotating OL. For a deep OL, we found that vortex nucleation occurs in a very different way from a usual rotating BEC in a harmonic trap. For the lattice spacing $d$ employed by Williams et al. [5], the motion of the density is frozen and vortices penetrate quickly from the outside through the density chinks. Local vortex nucleation, associated with the creation of vortex-antivortex pairs in the bulk region, occurs for lattice spacings larger than the critical value $d_{c}$. We also compare our numerical results with the experimental observations of Ref. [5], showing that the Gaussian envelope of the lattice beam is important in creating a difference in the scaling of the equilibrium vortex number on the rotation frequency.

\section{Acknowledgments}

We thank R. A. Williams and S. Al-Assam for helpful comments. The work of K.K. is partly supported by a Grant-in-Aid for Scientific Research from JSPS (Grant No. 21740267).
[1] I. Bloch, J. Dalibard, and W. Zwerger, Rev. Mod. Phys. 80, 885 (2008).

[2] N. R. Cooper, Adv. Phys. 57, 539 (2008).

[3] J. Dalibard, F. Gerbier, G. Juzeliunas, and P. Öhberg, arXiv:1008.5378 (2010).

[4] S. Tung, V. Schweikhard, and E.A. Cornell, Phys. Rev. Lett. 97, 240402 (2006).

[5] R. A. Williams, S. Al-Assam, C. J. Foot, Phys. Rev. Lett.
104, 050404 (2010).

[6] Y. Lin, R. L. Compton, K. J. Garcia, J. V. Porto, and I. B. Spielman, Nature(London), 462, 628 (2009).

[7] J.W. Reijnders and R.A. Duine, Phys. Rev. Lett. 93, 060401 (2004); Phys. Rev. A 71, 063607 (2005).

[8] H. Pu, L.O. Baksmaty, S. Yi, and N.P. Bigelow, Phys. Rev. Lett. 94, 190401 (2005).

[9] T. Sato, T. Ishiyama, T. Nikuni, Phys. Rev. A 76, 053628 
(2007).

[10] K. Kasamatsu, Phys. Rev. A. 79, 021604(R) (2009); J. Low Temp. Phys. 150, 593 (2007).

[11] D. S. Goldbaum and E. J. Mueller, Phys. Rev. A 77, 033629 (2008), 79; 063625 (2009); 79, 021602 (2009).

[12] M. P. Mink, C. M. Smith, and R. A. Duine, Phys. Rev. A 79, 013605 (2009).

[13] A. Trombettoni, A. Smerzi, and P. Sodano, New J. Phys. 7, 57 (2005).

[14] M. Polini, R. Fazio, A. H. MacDonald, and M. P. Tosi, Phys. Rev. Lett. 95, 010401 (2005).

[15] S. Sinha and Y. Castin, Phys. Rev. Lett. 87, 190402 (2001).

[16] M. Tsubota, K. Kasamatsu, M. Ueda, Phys. Rev. A 65, 023603 (2002); K. Kasamatsu, M. Tsubota, and M. Ueda, Phys. Rev. A 67, 033610 (2003).

[17] N. G. Parker, R. M. W. van Bijnen, and A. M. Martin, Phys. Rev. A 73, 061603(R) (2006)

[18] M. Yasunaga and M. Tsubota, J. Low Temp. Phys. 148, 363 (2007).

[19] S. Choi, S. A. Morgan, and K. Burnett, Phys. Rev. A 57, 4057 (1998). The value $\gamma=0.03$ was obtained by fitting a numerical simulation of the generalized GPE with the experimental data on collective excitations.

[20] A. A. Penckwitt, R. J. Ballagh, and C. W. Gardiner, Phys. Rev. Lett. 89, 260402 (2002).

[21] A. Griffin, T. Nikuni, and E. Zaremga, Bose-Condensed Gases at Finite Temperatures (Cambridge University Press, Cambridge, 2009);

[22] B. Jackson, J. F. McCann, and C. S. Adams, Phys. Rev. A 61, 013604 (1999).
[23] K.W. Madison, F. Chevy, V. Bretin, and J. Dalibard, Phys. Rev. Lett. 86, 4443 (2001).

[24] T. Frisch, Y. Pomeau, and S. Rica, Phys. Rev. Lett. 69, 1644 (1992); T. Winiecki, J. F. McCann, and C. S. Adams, ibid. 82, 5186 (1999).

[25] C. Raman, J.R. Abo-Shaeer, J.M. Vogels, K. Xu, and W. Ketterle, Phys. Rev. Lett. 87, 210402 (2001); B. M. Caradoc-Davies, R. J. Ballagh, and K. Burnett, ibid. 83, 895 (1999).

[26] A. L. Fetter, Rev. Mod. Phys. 81, 647 (2009).

[27] Intrinsically, $u_{2 \mathrm{D}}$ should be a nontrivial function of $V_{0}$ through the typical condensate size $R_{z}$ along $z$-axis, because the condensate can escape along the longitudinal $(z-)$ direction with increasing $V_{0}$, which leads to increase in $R_{z}$. Thus, one can expect that $u_{2} D$ would be decreased with $V_{0}$.

[28] R. A. Williams and S. Al-Assam (private communication).

[29] V. Schweikhard, S. Tung, and E. A. Cornell, Phys. Rev. Lett. 99, 030401 (2007).

[30] D. R. Scherer, C. N. Weiler, T. W. Neely, and B. P. Anderson, Phys. Rev. Lett. 98, 110402 (2007).

[31] P. B. Blakie, A. S. Bradley, M. J. Davis, R. J. Ballagh, C. W. Gardiner, Adv. Phys. 57, 363 (2008).

[32] S. P. Cockburn and N. P. Proukakis, Las. Phys. 19, 558 (2009).

[33] R. P. Feynman, Prog. Low Temp. Phys. 1, 17 (1955).

[34] S. Al-Assam, R. A. Williams, and C. J. Foot, Phys. Rev. A 82, 021604(R) (2010). 